\title{
Where cosmic rays come from?
}

\section{The class of $X$-ray stars which are also sources of gamma rays, one the object called Geminga, shows how much there is to do before they can be called the source of cosmic rays.}

IF there were ever any doubt of the value in astronomy of ancient if crude observations of phenomena, it would surely be dispelled by the demonstration in this week's issue (p. 464) of how Bignami, Caraveo and Paul have been able to use a Chinese record dating from $A D 437$ to narrow down the choice of a model for Geminga, the $y$-ray source. On the face of things, the tale resembles that of the identification of the Crab nebula as the remnant of a supernova observed by the Chinese a millennium ago, and recorded with sufficient precision for the identification to be convincing. The difference is that the star which surprised Chinese observers six centuries earlier is nowhere near a supernova remnant comparable with the Crab. That, so far as can be told, it lies close to the position now observed for Geminga proves nothing, but in the absence of other information and in the knowledge that Geminga must be an exceptional object, it is natural that people should put the two together.

Geminga itself requires more than casual explanation, as do the handful of other $\gamma$ ray sources now recognized. The obvious difficulty is that the $\gamma$-rays (whose spectrum extends above $50 \mathrm{MeV}$ ) are far more energetic than can be accounted for by thermal processes. With the rule of thumb that one electron-volt is roughly the equivalent of $10^{4} \mathrm{~K}, \gamma$-rays in this range could be generated only at temperatures of the order of $10^{12} \mathrm{~K}$. And the mechanism invoked for the generation of X-rays in binary systems, involving the infall of material, usually from a companion star, onto the surface of a neutron star, with or without the annihilation of positrons and electrons near the surface, is similarly inadequate.

Although Geminga was recognized as a $\gamma$-ray source a decade ago, it is only now that enough data have accumulated to say much about it. The first discoveries of $\gamma$ rays were made from satellites (but have since been repeated at a higher energy by ground-based detectors). In the past five years, an object within the same halfdegree region of the sky has been recognized also to be an X-ray source first by the Einstein satellite and, more recently, by Exosat. These are the data which now sustain the belief that Geminga is a periodic source of radiation with a period of almost exactly one minute. This was the development which led to the short burst of speculation, last year, that Geminga might be such an unusual object as to be respon- sible for exciting the minute-long natural oscillation of the Earth by means of gravitational radiation.

Mistakes like that are by no means unexpected, given the difficulty of extracting data from objects such as $\gamma$-ray stars. The problems are well illustrated by the account of Bignami et al. with two associates, also in this issue (p. 481), of the variability with time of the X-ray emission from Geminga. Nearly ten hours of satellite observation appear to have sent only 700 useful X-ray photons through the Exosat detectors. Direct measurement of, say, the energy spectrum is only on the margin of what is possible. Wringing an estimate of the periodic fluctuation of the radiation from such an object is an act of faith. Yet Bignami et al. derive not merely the period but also the rate at which it has increased.

The extraction of a period from such data is necessarily a statistical exercise, and not a particularly objective one. What Bignami et al. have done is to fit the arrival times of the small bunch of photons they have been able to extract from the published X-ray data to various periods in the neighbourhood of 59 seconds. In doing this, the authors have plainly been looking over their shoulders at the chequered history of the supposition that Geminga is indeed a periodic source of $X$-rays first put forward in 1977 but withdrawn in 1981. Pending the accumulation of more data, it is natural that Bignami et al. should take on trust the previous estimate of the period.

In such circumstances, model builders plainly enjoy uncomfortably many degrees of freedom. Bignami et al. consider but reject the possibility that the Geminga source is an isolated neutron star. Even if such an object were able to convert energy into high-energy photons efficiently, it would have to be implausibly close to the Solar System to function as observed. If, on the other hand, the period of 59 seconds is not that of the primary rotation of a neutron star but the precession of its axis, there should be a second shorter period of fluctuation (not found in the data) and "conventional" pulsar radiation should be much more powerful - but even now, no radio pulsations from Geminga have yet been observed.

The authors settle instead for a model put forward two years ago to account for $\gamma$-ray emission from the X-ray source Cygnus X-3, involving a binary system of which one member is a neutron star. To fit the optical observations, its companion in
Geminga must be a dwarf star. But how can such a system have a period of mutual rotation less than a minute long? The explanation, according to Bignami et al., is that the true period of orbital rotation is much longer, getting on for three hours, but that the neutron star component of the binary is the source of a beam of highenergy particles accelerated towards the more massive dwarf star and then converted by nuclear collisions into $\gamma$-rays and neutral pions. The period observed will thus be that corresponding to the beat frequency between the orbital and stellar rotation.

Precisely what should be made of this proposal is bound at this stage to be a matter of personal taste. With so few data, the remarkable feature of the situation is not that so much doubt persists but that anything worthwhile has been gleaned about Geminga. Moreover, there are now several testable proposals, of which the most intriguing may be the possibility that what seems to have been a once-and-for-all change in the rate of increase of the observed period may coincide with the surprising increase of the flux of cosmic $\gamma$ rays (with energies in excess of $10^{12} \mathrm{eV}$ ) early in 1980, well observed by groundbased detectors. A second coincidence would in the circumstances be almost clinching.

Of the puzzles that remain, the most puzzling is the origin of the object. Isolated neutron stars may be visualized easily enough as having begun their existence spinning much more quickly, and to have been progressively decelerated. If the period of fluctuation assigned to Geminga is indeed that corresponding to the beat frequency between two other rhythmic motions, it is a little easier to understand how the system may have been created comparatively recently. The possibility that the binary system was created in the collapse of an unstable massive star which somehow avoided a supernova explosion is attractive but, for the time being, untestable.

So why not wait until there are enough data to satisfy all reasonable curiosity? Because that will take many more X-ray satellites, and because the issue is potentially too important to be left in limbo. For there is a possibility that these objects are the principal source of cosmic rays in the galaxy. It would be a great convenience if that were the case.

John Maddox 